\title{
Acute organic brain syndrome due to drug-induced eosinophilia
}

\author{
Soo-Chin Ng' ${ }^{1}$ M.K. Lee ${ }^{2}$ and Alan Teh ${ }^{2}$ \\ ${ }^{1}$ Haematology Division, Department of Pathology and ${ }^{2}$ Department of Medicine, Medical Faculty, University of \\ Malaya, 59100 Kuala Lumpur, Malaysia.
}

\begin{abstract}
Summary: A 72 year old man developed acute organic brain syndrome associated with marked eosinophilia following self medication with a variety of drugs. Investigations revealed no other known causes of eosinophilia. Withdrawal of drugs resulted in dramatic drop in eosinophil count paralleled by clinical resolution of neurological problems. To our knowledge drug-induced eosinophilia has not previously been associated with acute organic brain syndrome.
\end{abstract}

\section{Introduction}

Self medication with multiple drugs is not an uncommon practice in the Far East. We report an unusual case of drug-induced eosinophilia presenting with acute organic brain syndrome which resolved with withdrawal of drugs.

\section{Case report}

A 72 year old male Chinese retired engineer was admitted to the University Hospital, Kuala Lumpur in January 1988 with absent-mindedness, unsteady gait and visual hallucination for the previous 6 weeks. These symptoms progressed insidiously as the patient became disorientated and talked irrelevantly. He subsequently required support to walk and became incontinent of urine. Prior to this illness he had been mentally agile, managing a consultant engineering firm until he retired 2 years previously. He had no complaints until 2 months previously when he developed chronic cough for which he treated himself with a variety of drugs (in a 'cocktail' fashion) which included ampicillin, amoxycillin, erythromycin, tetracycline, aspirin and paracetamol as well as Chinese and Siamese traditional medicine. He was a heavy smoker but did not take alcohol regularly. He was diagnosed to have pulmonary tuberculosis 40 years ago. Clinical examination revealed an alert but grossly disoriented patient. He was not agitated and speech was coherent but irrelevant. There was marked impairment of recent and remote memory. He did not

Correspondence: S.C. $\mathbf{N g}$, M.R.C.P. (UK).

Accepted: 25 April 1989 appear to be hallucinating. No focal neurological deficits were detected.

He was investigated as a case of acute organic brain syndrome. Skull radiographs were normal. Chronic subdural haematoma was excluded by computerized tomography which showed only diffuse cerebral atrophy. Electroencephalography was normal. Full blood counts revealed leucocytosis of $37.36 \times 10^{9} / 1$, with extreme eosinophilia (57\%) and an absolute eosinophil count of $21.30 \times 10^{9} / 1$. Erythrocyte sedimentation rate was markedly raised at $110 \mathrm{~mm}$ in the first hour. Bone marrow aspirate showed a normocellular marrow with prominent eosinophils and eosinophilic precursors constituting $35 \%$ of the nucleated cells. No increase in blast was noted. Trephine biopsy showed similar findings with no increase in fibrosis. Serum electrolytes were normal. Renal and liver functions were unremarkable. VDRL and TPHA tests were negative. Cysts and ova were absent on stool examination. Increased lung markings consistent with interstitial lung disease secondary to smoking were seen in the chest radiograph; screen for tuberculosis was negative. Echocardiography was normal. The serum immunoglobulins were as follows: IgG $1930 \mathrm{mg} / \mathrm{dl} ;$ IgM $819 \mathrm{mg} / \mathrm{dl} ;$ IgA $194 \mathrm{mg} / \mathrm{dl}$. Serum electrophoresis showed a mild diffuse increase in gamma globulins. Complement levels were normal. Antinuclear factor was not detected. Nerve conduction studies were within normal limits.

The patient was managed conservatively as druginduced eosinophilia. All previous medications were stopped. The patient's eosinophil count dropped sharply (Figure 1) and there was rapid improvement in cognitive functions. By the 7th hospital day he was orientated and able to answer simple questions. 


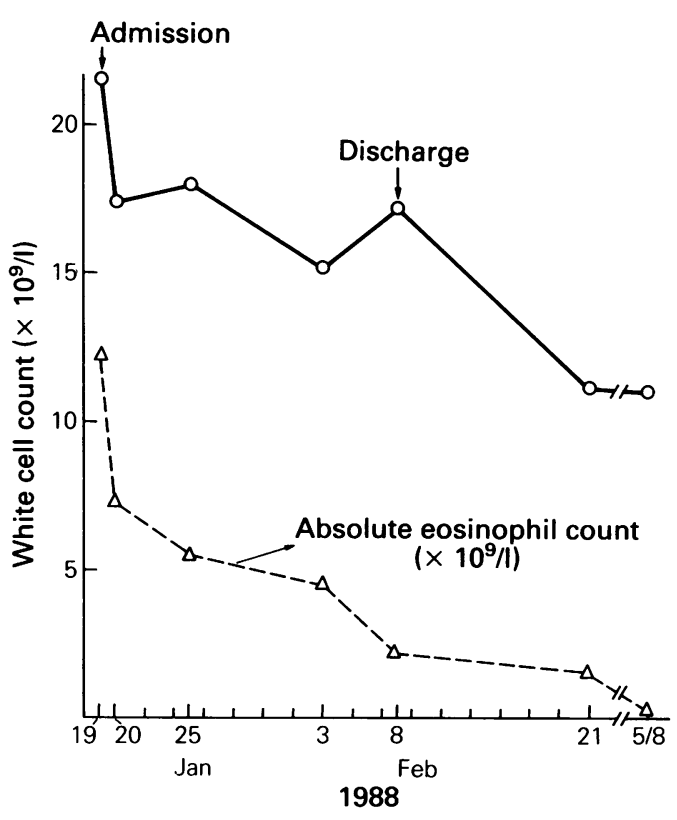

Figure 1 Clinical course of patient.

Memory showed progressive improvement too. At follow-up 7 months later, his cognitive functions were normal. His eosinophil count and erythrocyte sedimentation rate were within normal limits.

\section{Discussion}

Drug-induced eosinophilia is well known but otherwise rare, the incidence being estimated at less than

\section{References}

1. Spry, C.J.F. Eosinophilia and allergic reactions to drugs. Clin Haematol 1980, 9: 521-534.

2. Chusid, M.J., Dale, D.C., West, B.C. \& Wolff, S.M. The hypereosinophilic syndrome. Analysis of fourteen cases with review of the literature. Medicine 1975, 54: 1-27.

3. Fauci, A.S., Harley, J.B., Roberts, W.C., Ferrans, V.J., Gralnick, H.R. \& Bjornson, B.H. The idiopathic hypereosinophilic syndrome: clinical, pathophysiologic and therapeutic considerations. Ann Intern Med 1982, 97 78-92.
$0.1 \% .{ }^{1}$ However, a few drugs, such as gold, can cause eosinophilia in as many as half of the patients. ${ }^{1}$ In view of the prominent history of multiple drug ingestion by the patient over the preceding 2 weeks and the absence of other known causes of eosinophilia, our patient was managed as drug-induced eosinophilia and showed remarkable spontaneous clinical improvement in neurological function with concurrent exponential drop in absolute eosinophil counts. Owing to multiple drug ingestion, it was not possible to pinpoint the 'culprit' drug(s). Features suggestive of drug allergy such as fever, skin lesions, lymphadenopathy and splenomegaly were absent. Factors which might have predisposed to drug hypersensitivity reactions such as atopy, asthma, and slow drug clearance due to hepatic or renal disease were not found. Screening for other known causes of eosinophilia was negative.

Although neurological symptoms are well documented in patients with idiopathic hypereosinophilic syndrome, ${ }^{2,3}$ these have rarely been reported in association with eosinophilia in other diseases. ${ }^{4}$ Central nervous system dysfunction as the sole manifestation of eosinophilic reaction as seen in our patient has not been reported. In a comprehensive review of drug-induced eosinophilia by Spry, ${ }^{1}$ neurological proอ blems were not included as a clinical feature. Othef manifestations of drug-induced eosinophilia such as cardiovascular, pulmonary, haemopoietic, hepatic dermatological, gastrointestinal or renal involvement were not seen in this patient.

The central nervous system manifestations in th hypereosinophilic syndrome are thought to be relatef to neurotoxin present in human eosinophil granules which have been shown to cause demyelination and to damage Purkinje cells in laboratory animals. ${ }^{5}$ The mechanism of central nervous system dysfunction in drug-induced eosinophilia is not known.

4. Ravindran, M. Tropical eosinophilia presenting with neurological features. $\mathrm{Br}$ Med J 1979, 79: 1262.

5. Durack, D.T., Sumi, S.M. \& Klebanoff, S.J. Neurotoxicity of human eosinophils. Proc Natl Acad Sci USA 1979, 76: $1443-1447$. 